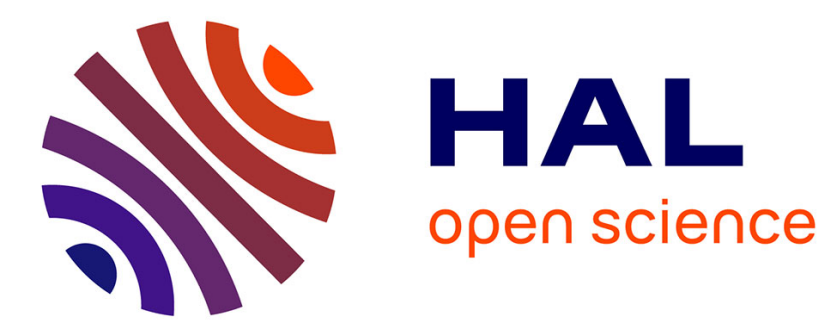

\title{
Experimental study of aerated cavitation in a horizontal venturi nozzle
}

\author{
Petar Tomov, Sofiane Khelladi, Florent Ravelet, Christophe Sarraf, Farid \\ Bakir, P. Vertenoeuil
}

\section{- To cite this version:}

Petar Tomov, Sofiane Khelladi, Florent Ravelet, Christophe Sarraf, Farid Bakir, et al.. Experimental study of aerated cavitation in a horizontal venturi nozzle. Experimental Thermal and Fluid Science, 2016, 70, pp.85-95. 10.1016/j.expthermflusci.2015.08.018 . hal-01176675v3

\section{HAL Id: hal-01176675 \\ https://hal.science/hal-01176675v3}

Submitted on 14 Jan 2020

HAL is a multi-disciplinary open access archive for the deposit and dissemination of scientific research documents, whether they are published or not. The documents may come from teaching and research institutions in France or abroad, or from public or private research centers.
L'archive ouverte pluridisciplinaire HAL, est destinée au dépôt et à la diffusion de documents scientifiques de niveau recherche, publiés ou non, émanant des établissements d'enseignement et de recherche français ou étrangers, des laboratoires publics ou privés. 


\title{
Experimental study of aerated cavitation in a horizontal venturi nozzle
}

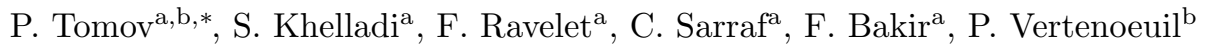 \\ ${ }^{a}$ DynFluid Laboratory, EA92, Arts et Métiers ParisTech \\ 151 Boulevard de l'Hôpital, 75013 Paris, France \\ ${ }^{b}$ SNECMA, SAFRAN group, \\ Rond-point Réné Ravaud, 77550 Réau, France
}

\begin{abstract}
The injection of bubbles into an already cavitating flow is a way of influencing the typical cavitating behavior. The present article deals with experiments on aerated and non-aerated cavitation in a transparent horizontal venturi nozzle. The observations are done by means of a high-speed camera. In such a way the extremely rapid cavitation and cavitation-aeration flows are captured and further analysed. The post-processing techniques is based on the detection of the grey level on the series of images. As a result, three different regimes are identified: sheet cavitation, cloud cavitation and "supercavitation". Those regimes are further aerated by injecting air bubbles. Standard deviations, timespace diagrams and frequency spectrum based on the vertical distribution of the grey level along a monitored line are plotted for all of the observed regimes. In the pure cavitation cases we obtain statistically symmetrical structures with characteristic lengths and frequencies. On the other hand, with aeration present, the symmetry is broken and characteristic lengths and frequencies are deeply modified, until a complete disappearance when "supercavitation" is reached. Keywords: Sheet cavitation, Cloud cavitation, Supercavitation, Aerated cavitation, Venturi nozzle
\end{abstract}

\footnotetext{
${ }^{*}$ Corresponding author

Email address: Petar.TOMOV@ensam.eu (P. Tomov)
} 


\section{Introduction}

Cavitation is a well known physical phenomenon occurring in various technical applications. When the pressure becomes inferior to the saturating vapour pressure of the liquid, cavitation takes place. The cavitation is responsible for issues like erosion (Fortes-Patella and Reboud, 1998; Fortes-Patella et al., 2013; Petkovšek and Dular, 2013), noise and vibrations (Tsujimoto et al., 2001; Tsujimoto, 2006), which can lead to a malfunctioning of various turbo-machines (Pouffary et al., 2008), for instance impellers (Mejri et al., 2006; Campos Amezcua, 2009). In general understanding, the cavitation occurrence has a negative effect on the proper functioning of a hydraulic system. However, in some particular cases, it can have an extremely positive effect leading to a drag reduction, as in the case of submarine vehicles (Wosnik and Arndt, 2013). In that case the supercavitating structure covers the immersed body and makes it slip through the liquid (Ceccio, 2010), which results in a extremely rapidly moving object. It is very important for one to be able to understand the physics behind the two-phase flow phenomenon, in order to reduce the negative effect or increase its positive influence. In that sense, studying cavitation dynamics in simple geometries like convergent-divergent venturi nozzles is a way of achieving that goal.

\subsection{Sheet cavity dynamics}

The sheet cavity dynamics has been widely studied in the case of a venturi nozzle (Stutz and Reboud, 2000; Decaix and Goncalvès, 2013; Chen et al., 2015), in the case of a hydrofoil (Kjeldsen et al., 2000; Dular et al., 2005, 2012) or on a divergent step (Callenaere et al., 2001). The dynamical characteristics of a hydrofoil change considerably with different angle of attack and cavitation number (Kjeldsen et al., 2000). In the case of the venturi nozzle, a periodic cycle can take place, which consists of the following steps. Firstly, the cavity grows from the venturi throat, secondly a re-entrant jet appears at the sheet cavity closure, flows upstream on the wall, and eventually cuts the newly formed 
vapour phase. In general, the re-entrant jet is created by the flow which expands in the closure region, in such a way, that in combination with the venturi wall, it creates a stagnation point. The conservation of momentum makes the fluid to pass beneath the cavity. As a result, the jet progresses and results in a vapour separation (Wade and Acosta, 1966), forming a cloud which is being further advected. The cloud vapour collapses in the divergent venturi nozzle zone where the pressure is substantially higher than the one at the throat. The cavity length is reduced and the whole process repeats itself. The repeatability of the process is characterized by the shedding frequency $f_{s}$. Some experiments have been done with colored water in order to reveal the dynamics of the re-entrant jet (Le et al., 1993). Other studies have shown that the adverse pressure gradient has a primary role in the development of the jet (Callenaere et al., 2001; Ceccio, 2010). Recently Ganesh, H. et al. (2014) showed that the shedding mechanism could also be governed by the shock wave in bubbly mixture and not by the re-entrant jet. The study of the dynamics of the re-entrant jet is beyond the scope of the present paper. Nevertheless, one could mention the possibility for different regimes to take place. For instance, when the pressure at the venturi throat is not sufficiently low, the sheet cavity length is not long enough for the cloud cavitation shedding to occur (Keil et al., 2011, 2012). In such kind of a regime the cavity oscillates and the cavity closure position does not shift in time. As a result, there are no large vapour clouds advected. The different cavitation regimes are characterized by different Strouhal numbers, which values are not universal (Coutier-Delgosha et al., 2006; Dular and Bachert, 2009; Sayyaadi, 2010), and have been recently studied with Proper Orthogonal Decomposition by Danlos et al. (2014b).

By its nature, the cloud cavitation has an extremely aggressive behavior and it is capable of doing severe damage on the solid surface (Fortes-Patella et al., 2000; Dular and Petkovšek, 2015). This is due to the extremely high pressure waves at the moment of bubbles' collapse. As a result, a control of the cavitation behavior can lead to a stable regime instead of having an unsteady damaging one. Some passive control methods based on surface roughness have 
been studied by Danlos et al. (2014a).

\subsection{Aeration}

Another recent technique capable of influencing the cavitation inception is the aeration of the cavitating liquid. Davis (2008) and Dunn et al. (2010) injected a controlled quantity of bubbles into a transparent venturi nozzle, in order to study its effects on the cavitation in the case of water and aviation jet fuel. They have found that the position of cavitation inception can be spatially shifted, if an injection of gas is to take place or not. Shamsborhan et al. (2010) measured the speed of sound in a two-phase flow, which was characterized by a high void fraction. In order to achieve such a high quantity of gas, an intrusive injection of air into a liquid flow has been done. Dong and Su (2006) presented an experimental investigation of cavitation control by aeration. The pressure waveforms were analysed with and without aeration. The results showed that the aeration phenomenon increases in a remarkable manner the pressure in the cavitation region and the corresponding pressure waves exhibit a shock wave. Aeration of a moving cavitating body can also result in "supercavitation" (Semenenko, 2001), for instance in Savchenko (2001) and Wosnik and Arndt (2013).

\subsection{Current study}

The work presented in the paper is part of an industrial project, which purpose is the study of the cavitation coupling with the out-gassing phenomenon at the inlet of a jet engine fuel pump. In order to deal with the complexity of the multiphase nature of the flow, an investigation on a simplified geometry is proposed, where the out-gassing effect is simulated by a controlled injection of air bubbles. From a scientific point of view, the purpose of the present study is twofold. Firstly, the double venturi nozzle geometry allows the observation and exploration of the symmetry of the sheet cavities at the top and bottom walls, their coupling under the influence of gravity and of the interaction between

the advected structures. Secondly, a controlled quantity of gas is injected into 
the already cavitating flow, resulting in a bubbly/plug flow. The cavitation behavior and its coupling with the air bubbles is observed by means of a highspeed camera. The images are post-processed to extract frequency spectrum and time-space diagrams, as well as standard deviation plots of the images gray level.

The article is organized as follows: the experimental set-up is firstly described in $\S 2$, followed by the images post-processing technique in $\S 3$. The results are presented and analysed in $\S 4$. Finally, concluding remarks are given in $\S 5$.

\section{Experimental Set-up}

The experiments were conducted in a closed loop test rig of the DynFluid Laboratory facilities (see Fig. 1). Two storage tanks of 150 liter capacity each provide water in the rig. The centrifugal pump moves the flow from tank 1 , which is always full, towards tank 2 . The cylindrical pipe has an inner diameter of $40 \mathrm{~mm}$. The discharge flow is monitored by a turbine flow meter $10 \mathrm{D}$ upstream of the air injection ring. The experimental venturi nozzle section is placed $7 D$ after the gas injection. A valve is placed before the second tank, in order to increase the pressure in the system if needed. It was left in open position during the experiments.

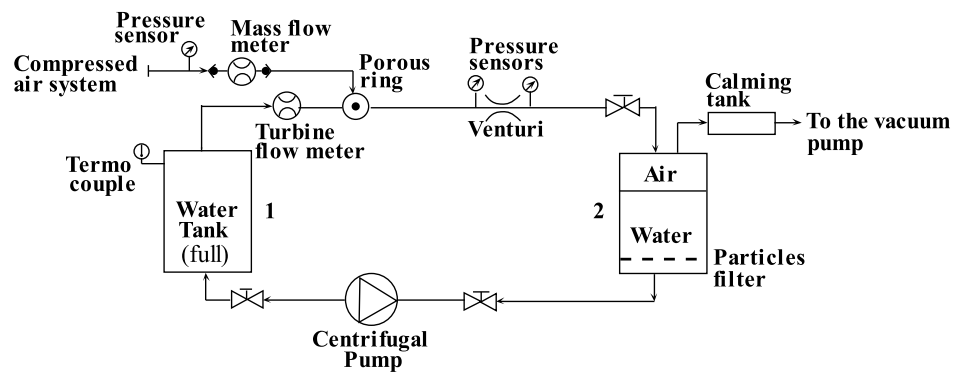

Figure 1: Sketch of the experimental set-up (not at scale)

The transparent horizontally symmetrical venturi profile is placed in the test section, between the tanks. The converging/diverging angles are $18^{\circ}$ and 
$8^{\circ}$, respectively. The inlet venturi height is $H_{\text {inlet }}=30 \mathrm{~mm}$ and its throat height is $H_{\text {throat }}=10 \mathrm{~mm}$, which gives an aspect ratio of 3 . The width is constant and equal to $10 \mathrm{~mm}$. The total length of the venturi test nozzle is $220 \mathrm{~mm}$. All positions in the test section are expressed with non-dimensional values $X^{*}=\frac{x}{H_{\text {throat }}}, Y^{*}=\frac{y}{H_{\text {throat }}}$ originating at the center of the throat section. Figure 2 is a sketch of the geometry of the venturi nozzle. It also shows the monitored line which will be discussed in details in the third and fourth part of the paper.

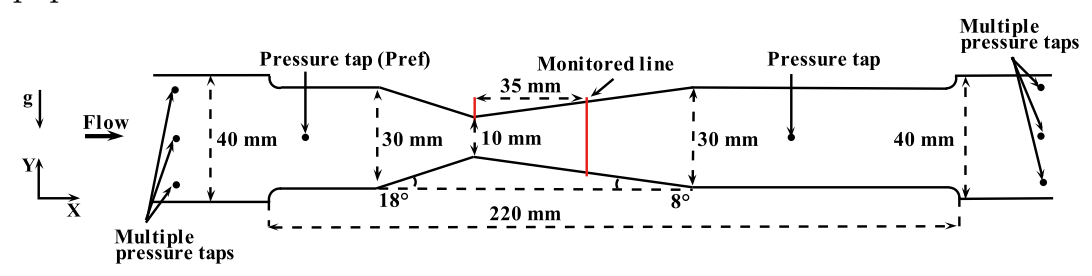

Figure 2: Venturi nozzle geometry (to scale)

The fluid motion is achieved by a Salmson centrifugal pump with a maximum flow rate capacity of $8 \mathrm{~m}^{3} / \mathrm{h}$. The centrifugal pump is placed on a lower level compared to the tanks in order not to suck in any vapor due to a possible outgassing in tank number 2. It is off-centered and fixed, in order not to produce any vibrations during the tests. Moreover, the upper horizontal part of the ducts are balted to a support, independently built between the two rig tanks. In such a way any vibrations coming from the liquid out-gassing in the tanks are overcome. In order to be able to decrease the pressure at the free surface while experiments are running, a vacuum pump is connected to the second tank.

\subsection{Measuring Instruments}

The pressure measurements have been done by two Jumo absolute pressure sensors mounted on the inlet and outlet sections at $X^{*}=-6$ ( 0 to 4 bar) and $X^{*}=10$ (0 to 2 bar), respectively. The response time of both of them is less than $3 \mathrm{~ms}$. The pressure taps have a diameter of $1.25 \mathrm{~mm}$ in order to avoid any flow disturbances inside the venturi nozzle. The absolute pressure measured at $X^{*}=-6$ is the reference pressure $P_{r e f}$ for the calculation of the cavitation 
number. An absolute pressure sensor is mounted on tank 2 , where the vacuum pump is connected. The range of working values is from 0 to 2 bar.

In the following, the relative uncertainties that are given are all based on the reading of the values. In order to monitor the temperature of the working fluid, a Guilcor $\mathrm{K}$ type thermocouple is mounted on tank 1. During all the experiments the temperature of the fluid has been monitored and is equal to $19^{\circ} \mathrm{C} \pm 1{ }^{\circ} \mathrm{C}$.

The discharge flow rate was constantly monitored via a Heliflu TZN turbine flow meter, with $10 D$ straight pipe upstream and $5 D$ downstream. The latter is calibrated for a working range of viscosities in the interval $0.6 \times 10^{-3}$ to $1.0 \times 10^{-2}$ Pa.s. The relative volume flow rate uncertainty is $\frac{\Delta Q_{l i q}}{Q_{l i q}}= \pm 0.08$.

The air injection is achieved by a compressed air system. In order to ensure proper and controlled injection of the bubbles, two air filters AW30 have been mounted before and after the air mass flow rate meter. The filtration is equal to $5 \mu \mathrm{m}$ and the maximum pressure is $1 \mathrm{MPa}$. The air injection is achieved in a non-intrusive manner by means of a porous ring made of sintered bronze tube (BLR) with an inner diameter equal to the inner diameter of the pipe. The size of the pores ranges from 13 to $90 \mu \mathrm{m}$, given by the manufacturer (Sintertech). The length of the sintered bronze ring is equal to $45 \mathrm{~mm}$ and its thickness is equal to $3 \mathrm{~mm}$. The air gas injection holes are equally spaced at $90^{\circ}$. The relative mass flow rate uncertainty is $\frac{\Delta M_{g a z}}{M_{g a z}}= \pm 0.05$, based on the screen readings. It has been checked, that the turbine flow meter, as well as the nonintrusive injection approach, do not generate any detectable flow perturbations within the accuracy of the instrumentation used in front of the venturi nozzle. The upstream fluid flow does not contain any bubbles, as a result of an early cavitation or out-gassing phenomenon.

The cavitation number is defined as $\sigma=\frac{\left(P_{\text {ref }}-P_{v a p}\right)}{\left(\frac{1}{2} \rho V_{r e f}^{2}\right)}$. The reference pressure $P_{r e f}$ is measured at the inlet of the venturi section at $X^{*}=-6$. The reference velocity $V_{\text {ref }}$ is the superficial velocity of water at the venturi throat. The values of $\sigma$ would be at most 3\% lower, if the pressure at the throat would have been taken as a reference pressure value. The inlet discharge velocity is calculated 
as a function of the flow rate. The vapor pressure in operating conditions is considered to be $P_{\text {vap }} \simeq 2200 \mathrm{~Pa}$ at $19 \pm 1^{\circ} \mathrm{C}$. In the article the temperature will be taken as $19^{\circ}$, which gives a relative uncertainty of $\frac{\Delta P_{v a p}}{P_{\text {vap }}}= \pm 0.06$.

The table 1 summarizes the relative uncertainties of the measured values $\frac{\Delta U}{U}$, as well as the impact on the calculated cavitation number.

\begin{tabular}{|c|c|c|c|c|c|c|}
\hline & $\mathbf{P}_{\text {vap }}$ & $\mathbf{P}_{\text {ref }}$ & $\mathbf{V}_{\text {ref }}$ & $\mathbf{M}_{\text {gaz }}$ & $\beta=\frac{\mathbf{Q}_{\text {gas }}}{\mathbf{Q}_{\text {gas }}+\mathbf{Q}_{\text {liq }}}$ & $\sigma$ \\
\hline$\pm \frac{\Delta U}{U}$ & 0.06 & 0.05 & 0.08 & 0.05 & 0.16 & 0.22 \\
\hline
\end{tabular}

Table 1: Relative uncertainties of the measured and calculated values

\subsection{Cavitation image capturing}

The transparent square nature of the venturi nozzle allows the use of a highspeed camera to make three-dimensional visualizations of the flow. In all of the experiments only side views are analysed. The camera is mounted on a tripod in order not to have any field capturing perturbations as a result of vibrations, coming from the pump or the out-gassing phenomenon. In order to visualize the sheet cavity, 4096 images at 1000 frames per second are acquired for each case study. A CamRecord 600 camera with a $100 \mathrm{~mm}$ Zeiss Makroplanar objective lens is used. The parameters used for the visualizations are presented in table 2 . The spatial resolution is $13 \mathrm{pix} / \mathrm{mm}$.

\begin{tabular}{|l|l|}
\hline Camera Charecteristcs & Value \\
\hline Focal length & $100 \mathrm{~mm}$ \\
Resolution & $1280 \times 512$ pixels \\
Acquisition rate & $1000 \mathrm{fps}$ \\
Exposure time & $1 / 25000 \mathrm{~s}$ \\
Pixel size & $12 \mu \mathrm{m} \times 12 \mu \mathrm{m}$ \\
Active area & $10.24 \mathrm{~mm} \times 8.19 \mathrm{~mm}$ \\
\hline
\end{tabular}

Table 2: Visualization Parameters of CamRecord 600 mounted with 100 mm Zeiss Makroplanar objective 
The flow is continuously illuminated from the backside by means of a Super Long Life Ultra Bright (SLLUB) White Led Backlight from Phlox. The light output area is a rectangular section of $200 \mathrm{~mm}$. The SLLUB minimal luminance is $3000 \mathrm{~cd} . \mathrm{m}^{-2}$. In all the case studies the output power has been kept constant equal to $99 \%$ of the maximal power of the backlight.

\section{Images post processing technique}

The post processing technique used in this study is based on the detection of the grey level on the images.

The first step of the post-processing technique consists of normalizing all instantaneous images $I$ by a reference image $I_{r e f}$. The latter is taken at a non-cavitating and not aerated flow. The process of normalization consists of a calculation of $I_{N}=\frac{I-I_{r e f}}{I_{r e f}}$.

The time averaged value of the grey level could be interpreted as the percentage of the number of frames where cavitation phenomenon is to take place at a particular spatial location, and the corresponding standard deviation values can be an indicator of the locations where any unsteady cavitation is present (Gavaises et al., 2015). The longitudinal characteristic length of the sheet cavity shedding is defined at the maximum value of the standard deviation as in Danlos et al. (2014b).

A value of 0 for the grey level corresponds to pure liquid phase, and a value of -1 would correspond to a region that completely absorbs the light. After the normalization step, a region of interest (ROI) is chosen. The ROI can be a straight line in any direction or a rectangular section. In the present study a straight vertical line is chosen (see Fig. 2) and time-space diagram of the grey level along the line is then analyzed.

Obvious peaks in a frequency spectrum may be generally related to spatial structures, with some characteristic dimension. The issue of universal definition of a Strouhal number in cavitation is not straightforward. This point has been summarized, discussed and a proposal for its unification has been given by Dular 
and Bachert (2009). In the present paper the Strouhal number is defined as $S t r_{L}=\frac{f_{s} \cdot L}{V_{r e f}}$ with $f_{s}$ being the highest frequency peak and $L$ is the longitudinal characteristic length, measured on the standard deviation images, as in Danlos et al. (2014b).

\section{Experimental results}

Two sets of experiments have been carried out and outlined in this paper. In the first case only cavitation phenomenon has been studied in the venturi nozzle. In the second case, the coupling between the cavitation and aeration has been shown. In the two cases the flow rate has been kept constant and the pressure at the free surface inside the second tank has been changed via the vacuum pump. The Reynolds number $R e=\frac{V_{\text {ref }} \cdot H_{\text {throat }}}{\nu}$ at the venturi throat is based on the reference velocity and the throat height. It is kept constant for all the experimental case studies. The gas mass flow rate is kept constant during the tests of aeration. The results will be presented in two separate subsections. In order to easier distinguish the case studies, all of the pure cavitating cases are referenced as (a), (b), (c), and all of the aerated cavitation cases are referenced as $\left(a^{\prime}\right),\left(b^{\prime}\right)$ and $\left(c^{\prime}\right)$.

\subsection{Cavitation results}

\begin{tabular}{|c|c|c|c|c|c|}
\hline Case & $\begin{array}{c}\mathbf{V}_{\text {ref }} \\
\left({\left.\mathrm{m} . \mathrm{s}^{-1}\right)}\right.\end{array}$ & $\mathbf{R e}_{\text {throat }}$ & $\begin{array}{c}\mathbf{P}_{\text {ref }} \\
(\mathrm{bar})\end{array}$ & $\sigma_{\mathbf{X}^{*}=-\mathbf{6}}$ & $\sigma$ \\
\hline (a) & 12.03 & $1.2 \times 10^{5}$ & 1.26 & 15.39 & 1.71 \\
(b) & 12.03 & $1.2 \times 10^{5}$ & 1.08 & 13.14 & 1.46 \\
(c) & 12.03 & $1.2 \times 10^{5}$ & 0.94 & 11.34 & 1.26 \\
\hline
\end{tabular}

Table 3: Cavitation experiment conditions

In the following paragraph the experimental cavitation results are presented. Table. 3 summarizes the conditions at which the experiments have been carried 

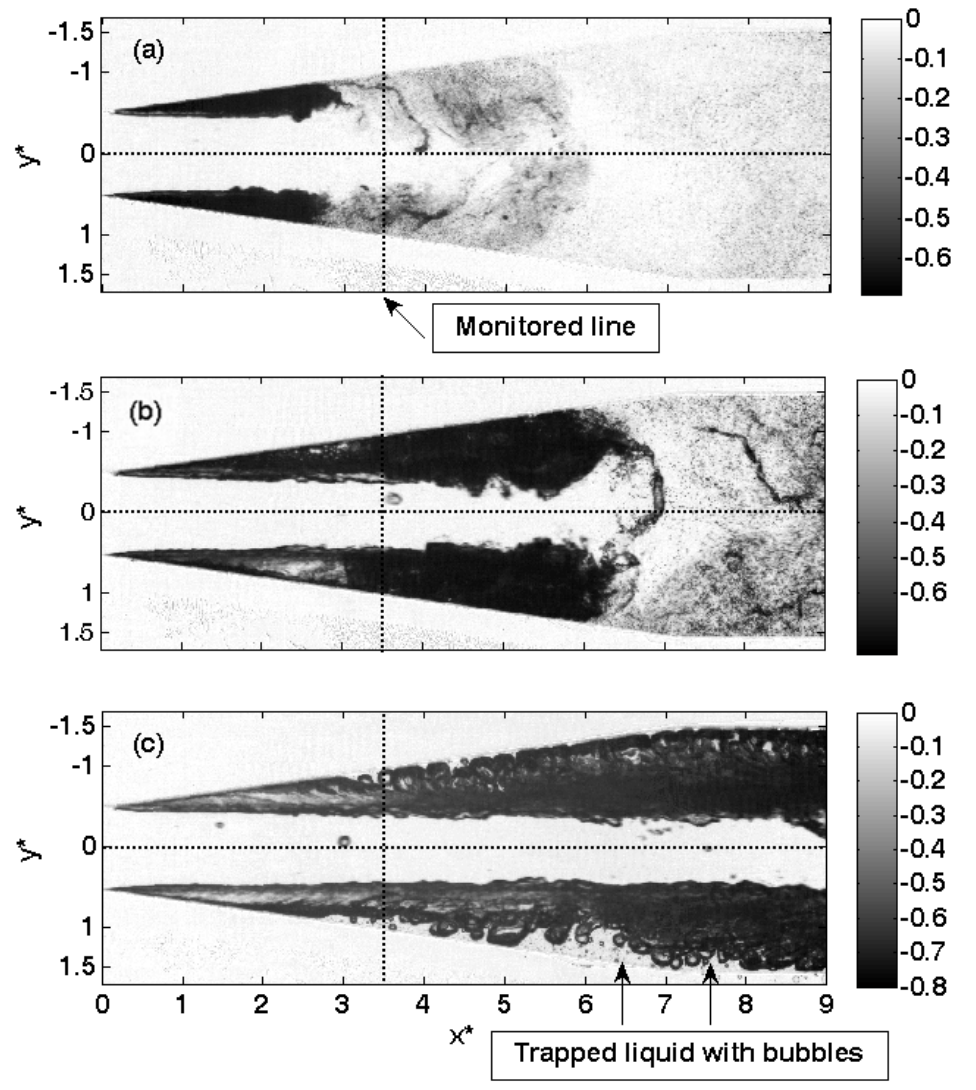

Figure 3: Normalized instantaneous grey image cavitation: (a) $\sigma=1.71$; (b) $\sigma=1.46$; (c) $\sigma=1.26$. The monitored line is at $X^{*}=3.5$ along which the time-space diagrams are plotted.

out.

Normalized grey instantaneous images taken from each case (cavitation at $\sigma=1.71$ (a), cavitation that tends to a "supercavitation" mode at $\sigma=1.46$ (b) and supercavitation $\sigma=1.26$ (c)) are shown in Fig. 3. The white color corresponds to a pure liquid phase and the grey values to the intensity of light absorption by the vapour phase. A time sequence of eight images is shown in Fig. 4. The standard deviations computed on the 4096 images are shown in Fig. 5. The time-space signal along the monitored line and FFT of it are shown in Fig. 6 . The axis in all the figures are normalized by $H_{\text {throat }}$, except the time space diagrams in Fig. 6, where the horizontal axis is the time, given in seconds. 
The monitored vertical line at $X^{*}=3.5$ is shown in Fig. 3. The horizontal symmetry lines are also drawn in Figs. 3 and 5 for eye-guiding purpose.

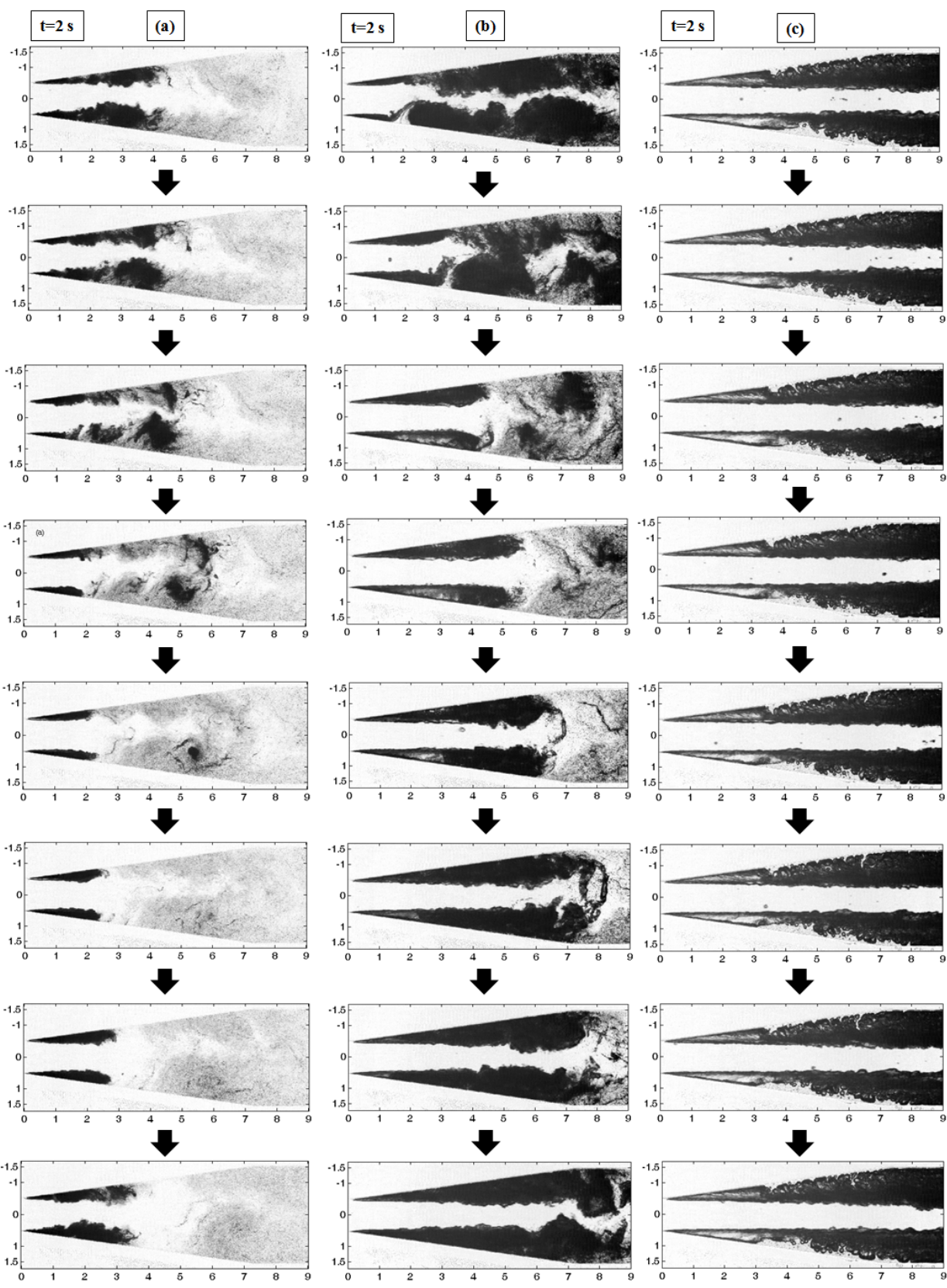

Figure 4: Sequence of images for cavitation starting at $t_{0}=2 \mathrm{~s}$, with a time step $\Delta t=10^{-3} \mathrm{~s}$ : (a) $\sigma=1.71$; (b) $\sigma=1.46$; (c) $\sigma=1.26$. 

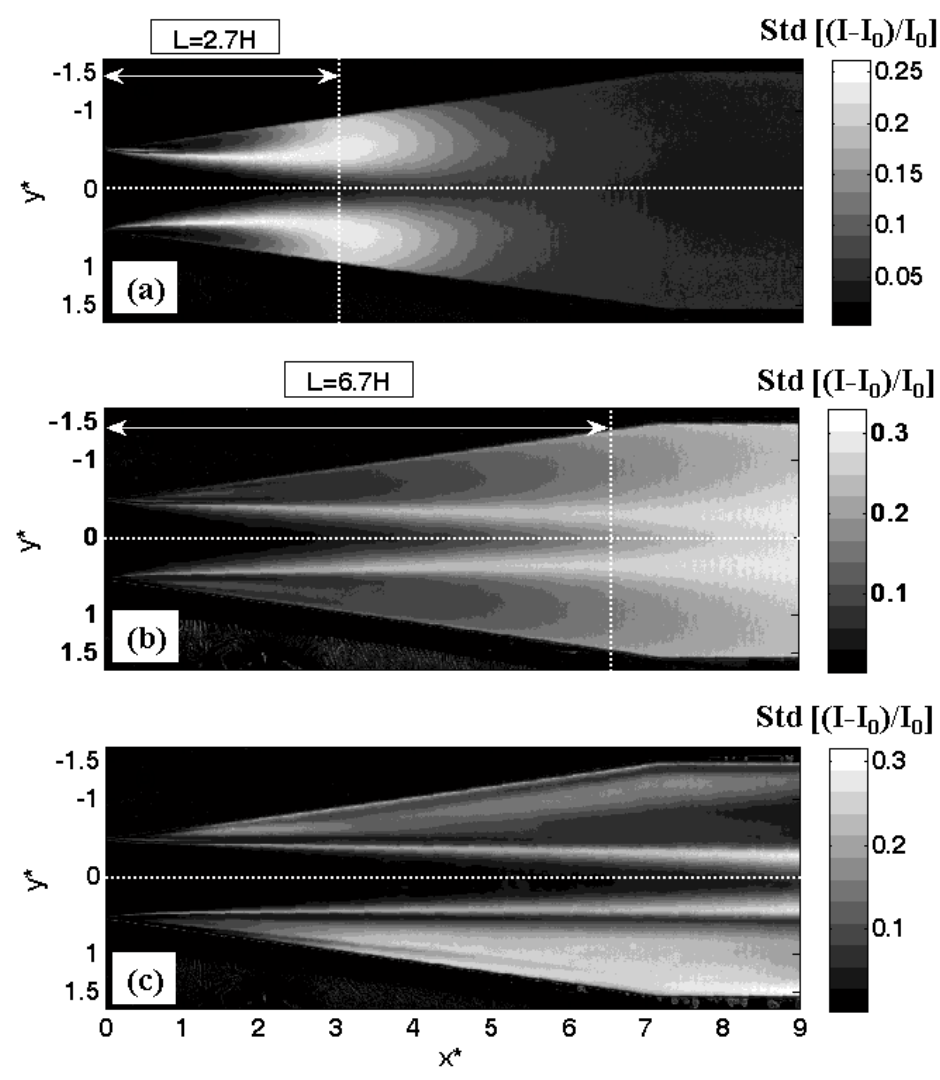

Figure 5: Standard deviation cavitation: (a) $\sigma=1.71$; (b) $\sigma=1.46$; (c) $\sigma=1.26$.

\subsubsection{Case study (a): cavitation at $\sigma=1.71$}

The instantaneous snapshot in figure 3(a) reveals the presence of two cavitation sheets on both sides, that seem to be very similar. The latter is confirmed by the symmetry of the standard deviation plot of the vapour phase distribution with respect to the $\overrightarrow{\mathbf{x}}$ axis in Fig. 5(a). A characteristic length of the cavitating vapour cloud is extracted from the standard deviation displayed in Fig. 5(a). This length is equal to $2.7 H_{\text {throat }}$. The cavitating sheet is indeed periodically cut in the vicinity of $2.7 H_{\text {throat }}$ by the re-entrant jet on both sides of the venturi, as can be seen in the time sequence of images (Fig. 4a), showing the dynamics of one cycle. The time-space diagram in Fig. 6(a) shows that the cavitation zones 
are in phase. At the moment of cloud cavity separation along the divergent part, the pressure gradient is responsible for the break up of the cloud into very small bubbles, that can be seen for instance in Fig. $3(\mathrm{a})$ between $X^{*}=3 H_{\text {throat }}$ and $6 H_{\text {throat }}$ lengths.

\subsubsection{Case study (b): cavitation at $\sigma=1.46$}

The instantaneous snapshot in Fig. 3(b), is very similar to the previous case, with two cavitation sheets on the top and bottom walls, which are almost twice larger. They are connected with a structure that resembles a hairpin vortex. The time sequence in Fig. 4(b) reveals a much more complex dynamics. On the first snapshot, one can see a cloud separation starting on the bottom sheet, around $X^{*}=2$, which triggers the cloud separation on the top wall. The two sheets then grow and bound together to give the situation of Fig. 3(b) at the middle of the time sequence, and grow further downstream up to the end of the diverging part: on the last image, they extend past $X^{*}=9$. The resulting regime is still statistically symmetric with respect to the $\overrightarrow{\mathbf{x}}$ axis as can be seen in Fig. 5(b). A characteristic length of $6.7 H_{\text {throat }}$ can also be extracted from Fig. 5(b), close to the symmetry axis and may statistically correspond to the presence of the hairpin vortex.

\subsubsection{Case study (c): "supercavitation" at $\sigma=1.26$}

When $\sigma$ is further reduced, the cavitating behavior tends to a "supercavitation", which can be seen in the instanteneous image in Fig. 3(c) and in the snapshots in Fig. 4(c). The two cavities fill the whole venturi nozzle, the cloud separations have disappeared resulting in the absence of any characteristic axial distance in the standard deviation plot in Fig. 5(c). As a result, a continuous liquid-vapour interface is created on both sides of the nozzle. Due to the convergence of the inlet section, the velocity at the venturi throat is of the order of $12 \mathrm{~m} \cdot \mathrm{s}^{-1}$, hence any further restrictions downstream, due to the large vapour presence, would only accelerate the liquid. Interestingly enough, a counter-flowing liquid pocket manages to penetrate upstream on both nozzle 
walls, as can be seen in Fig. 4(c), with more intensity on the bottom wall. This result in different levels of standard deviation between the two cavitating zones (Fig. 5(c)). Those flow disturbances are not entirely in phase and are not symmetrical, as it will be seen and discussed in the next section of the paper. The observed "supercavitation" is clearly not statistically symmetrical with respect to the horizontal axis.

\subsubsection{Cavitation time-space diagrams and frequency spectrums}

For case (a), one can see a dominant peak at $145 \mathrm{~Hz}$ in Fig. 6(a). The corresponding Strouhal number is $\operatorname{Str}_{L}=0.328$ for the characteristic length of $2.7 H_{\text {throat }}$. The obtained value corresponds to the interval between 0.2 and 0.4 proposed by Coutier-Delgosha et al. (2006). One can observe on the timespace diagram in Fig. 6(a) that the events are symmetrical and in phase on both sides of the venturi nozzle, which confirms the feeling when looking at the instantaneous images on the high-speed camera.

The behavior changes drastically for case (b), as can be seen in the timespace diagram in Fig. 6(b). It can be seen a symmetrical grey level spatial distribution on both sides of the venturi nozzle. The events that are pointed out by the textbox "cavity cloud separation" are statistically symmetrical with respect to the $\mathbf{x}$ axis and are in phase. The huge quantity of vapour present inside the nozzle results in a different frequency response, as it become clear in Fig. 6(b). The zone between the yellow lines in Fig. 6(b) points out the interface between the liquid and the cavitation sheets. One can notice the presence of peak values of the grey level along this interface. They correspond to a shedding on the top and bottom sides. This is the result of propagating instabilities at the liquid-vapour interface. The zone is turbulent and any small detached vapour structure causes an unstable behavior. The frequency analyzis supports the observations that as a result of the pressure decrease, the vapour phase is stretched downstream the divergent nozzle. Nevertheless, some cloud

separations take place at a frequency of $25 \mathrm{~Hz}$ with a corresponding $\operatorname{Str}_{L}=0.140$ for a length of $6.7 H_{\text {throat }}$. 

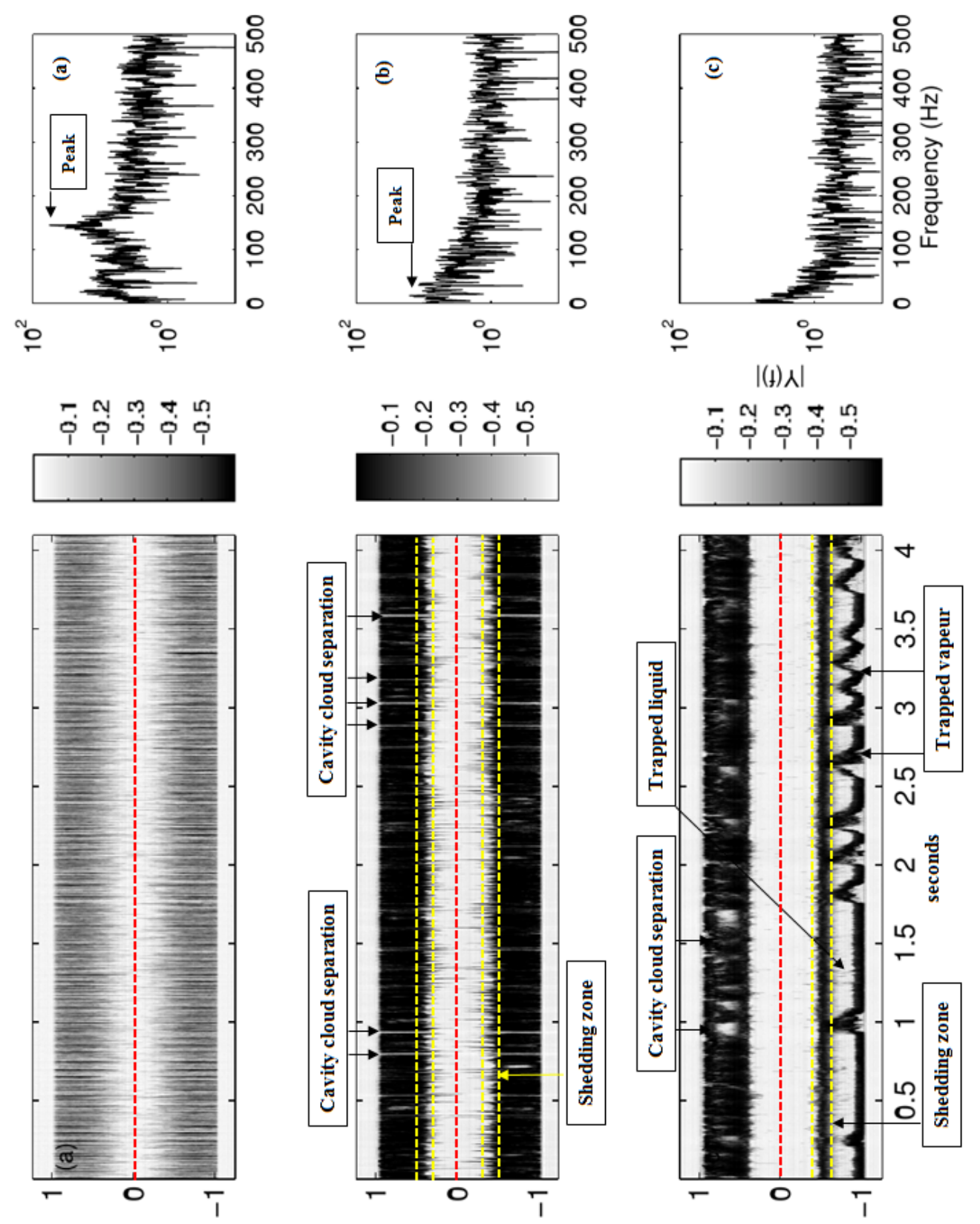

Figure 6: Cavitation time-space diagrams and frequency spectrum along vertical line at $x^{*}=$ 3.5: (a) $\sigma=1.71$; (b) $\sigma=1.46$; (c) $\sigma=1.26$. 
The time-space diagram for the "supercavitating" mode (c) is presented in Fig. 6(c). Semenenko (2001) has observed such a phenomenon on a range of similar velocities. There are no peaks, but a rather intense low frequency part in the spectrum. The overall behavior is similar to the one in Fig. 6(b), except the lack of a clearly distinguishable peak. Nevertheless, a frequency value of $7 \mathrm{~Hz}$ would correspond to the value of $25 \mathrm{~Hz}$ in case (b). On the other hand, the timespace diagram is quite different. On the top wall, one can observe an almost continuous vapour phase, except few entrapped liquid zones, which is not the case on the bottom venturi wall. On the bottom part, there is a thin continuous dark zone that is bounded by the yellow lines and labelled "shedding zone". On the bottom of this dark zone, one can clearly see a region with trapped liquid. Along the bottom wall, one can see a dark zone, with few lighter zones, that correspond to liquid flowing upstream. The transverse dark wavy forms are the result of trapped bubbles inside the trapped liquid. Due to the buoyancy force they manage to reach the shedding zone and are being advected by the high-speed flow. One can clearly identify the instances at $t=0.25 \mathrm{~s}$, in the vicinity of $t=1 \mathrm{~s}, t=2 \mathrm{~s}$ and $t=2.5 \mathrm{~s}$. Eight successively taken images at $t=2 \mathrm{~s}$ illustrate the instabilities in "supercavitation" regime in Fig. 4(c). Another observation is the very few instabilities at the liquid-vapour interface zone on either side, compared to Fig. 6(b). As a result, the amplitude decreases and there are no peaks. Table 4 summarizes the results for pure cavitation.

\begin{tabular}{|c|c|c|c|}
\hline Case & $\sigma$ & $\operatorname{Str}_{L}$ & $\begin{array}{c}\mathbf{F} \\
(H z)\end{array}$ \\
\hline (a) & 1.71 & 0.328 & 145 \\
\hline (b) & 1.46 & 0.140 & 25 \\
\hline (c) & 1.26 & - & - \\
\hline
\end{tabular}

Table 4: Cavitation results data table 


\subsection{Aerated cavitation results}

In this section we present the aerated cavitation experimental results. The flow velocity and the free surface pressures are kept equal to the ones in the previous section, in order to reveal the influence of the injected air over the cavitation phenomenon. A constant mass flow-rate of air is injected at the reference pressure. The corresponding values of the delivered volume concentration $\beta$ and of the cavitation number are given in table 5 .

\begin{tabular}{|c|c|c|c|c|c|c|c|}
\hline Case & $\begin{array}{c}\mathbf{M}_{\text {gas }} \\
\left(\mathrm{kg} . \mathrm{h}^{-1}\right)\end{array}$ & $\begin{array}{c}\beta=\frac{\mathbf{Q}_{\text {gas }}}{\mathbf{Q}_{\text {tot }}} \\
(\%)\end{array}$ & $\begin{array}{c}\mathbf{V}_{\text {ref }} \\
\left({\left.\mathrm{m} . \mathrm{s}^{-1}\right)}\right.\end{array}$ & $\mathbf{R e}_{\text {throat }}$ & $\begin{array}{c}\mathbf{P}_{\text {ref }} \\
(\mathrm{bar})\end{array}$ & $\sigma_{\mathbf{X}^{*}=-6}$ & $\sigma$ \\
\hline$\left(\mathrm{a}^{\prime}\right)$ & 0.072 & 9.6 & 12.03 & $1.2 \times 10^{5}$ & 1.33 & 15.66 & 1.74 \\
$\left(\mathrm{~b}^{\prime}\right)$ & 0.072 & 10 & 12.03 & $1.2 \times 10^{5}$ & 1.27 & 14.85 & 1.65 \\
$\left(\mathrm{c}^{\prime}\right)$ & 0.072 & 11.5 & 12.03 & $1.2 \times 10^{5}$ & 1.12 & 13.05 & 1.45 \\
\hline
\end{tabular}

Table 5: Aerated cavitation experimental conditions

The gas flow rate is a compromise between a quantity that can seriously influence the cavitating vapour structures and at the same time to be properly visualized on the high-speed camera images. A rough estimation of the injected

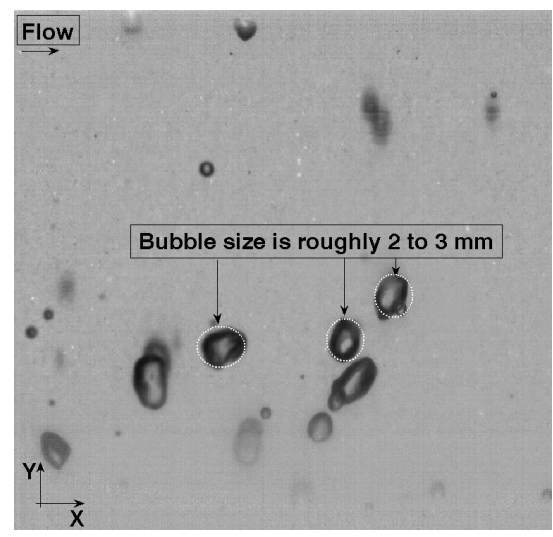

Figure 7: Rough estimation of the bubbles' sizes for aerated cavitation case (a') $\sigma=1.74$ on an instantaneous normalized image 
bubble's sizes is presented in Fig. 7 for the aerated cavitation (a'). Moreover, one can estimate the bubble throat size by observing Fig. 8, where the middle plane divides the venturi throat. The topology of the multiphase flow depends on the fluid properties and their superficial velocities. In the present case, according to Taitel and Dukler (1976) the observed regime lies between a bubbly and a plug flow. Despite the decrease of the free surface pressure inside the second water tank and the injection at the same time of air into the circuit, the inlet static pressure is measured higher than the one in pure cavitating mode: $\Delta P_{a^{\prime} \rightarrow a}=0.07$ bar, $\Delta P_{b^{\prime} \rightarrow b}=0.19$ bar and $\Delta P_{c^{\prime} \rightarrow c}=0.19$ bar. At the same time, the visually observed physical behavior is quite different, as it will be explained in the following sections.

\subsubsection{Aerated cavitation images}

As in the pure cavitation cases, normalized instantaneous snapshots of the aerated flows are shown in Fig. 8. A time sequence of eight images for each

aerated regime is displayed at $t=2 \mathrm{~s}$ in Fig. 9. The plots of the standard deviations are shown in Fig. 10. 

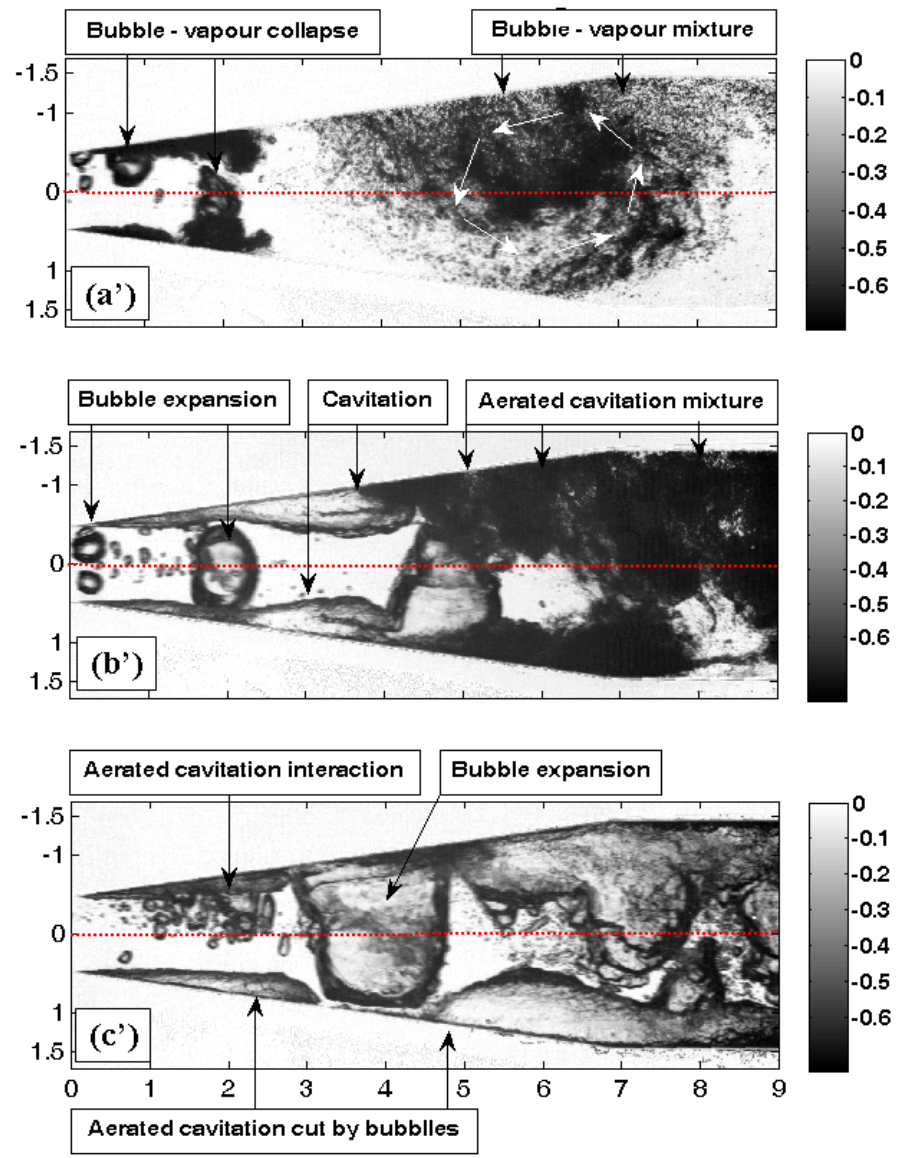

Figure 8: Normalized instantaneous grey image aerated cavitation: (a') $\sigma=1.74$; (b') $\sigma=$ 1.65; (c') $\sigma=1.45$. 

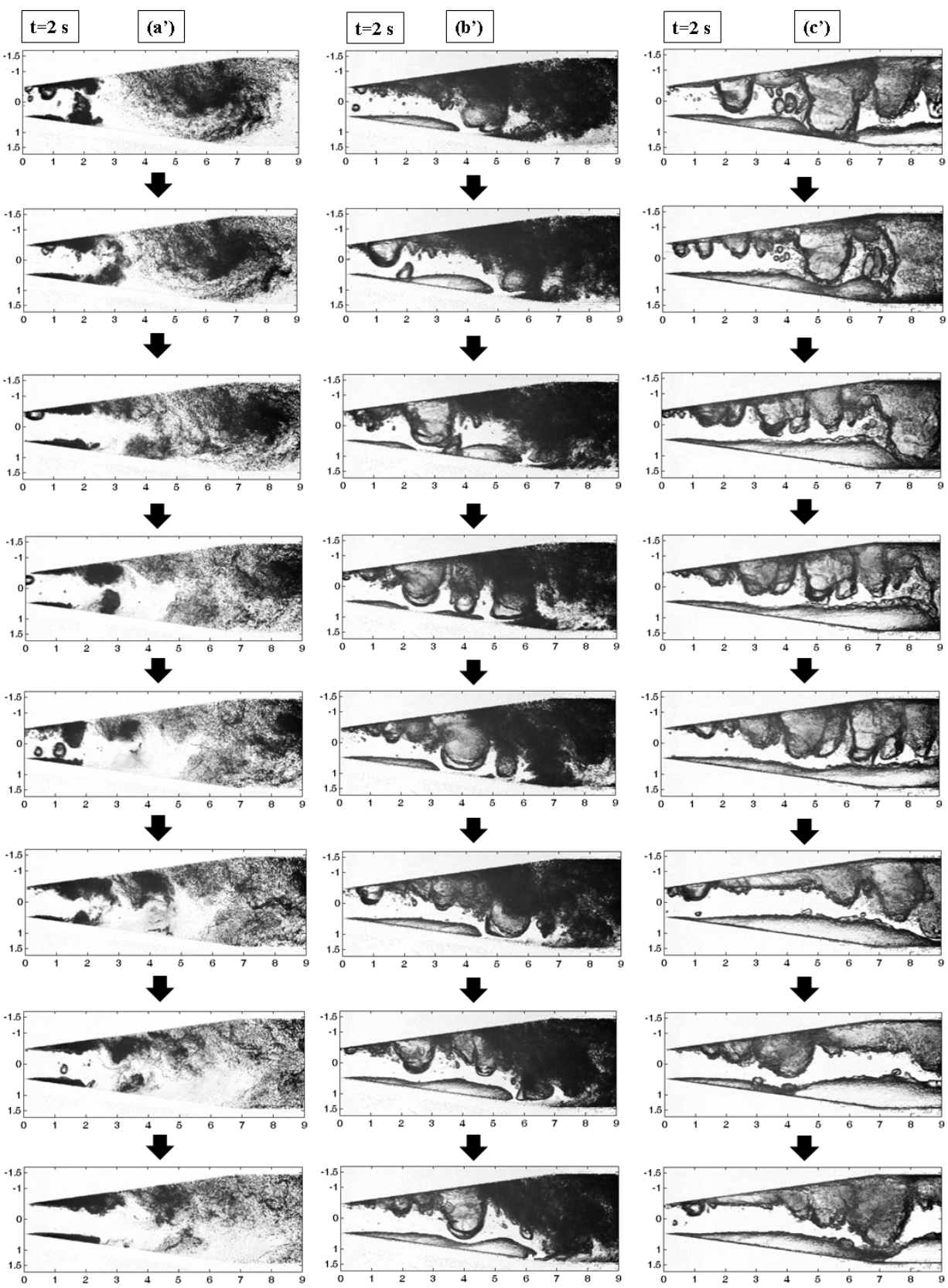

Figure 9: Sequence of images for aerated cavitation starting at $t_{0}=2 \mathrm{~s}$, with a time step $\Delta t=10^{-3} \mathrm{~s}:\left(\mathrm{a}^{\prime}\right) \sigma=1.74$; (b') $\sigma=1.65$; (c') $\sigma=1.45$. 

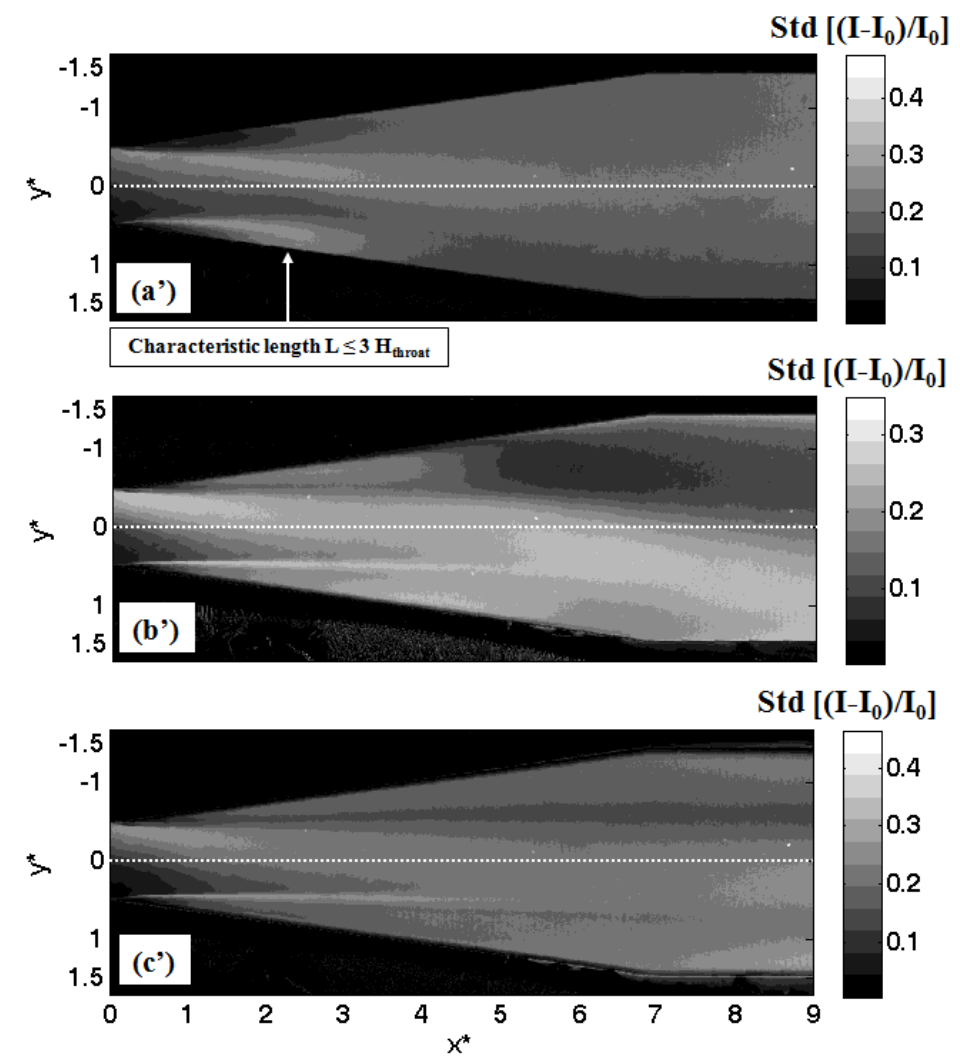

Figure 10: Standard deviation aerated cavitation: (a') $\sigma=1.74$; (b') $\sigma=1.65$; (c') $\sigma=1.45$.

The parameters for case (a') are very close to those for case (a). One can still observe two small cavitation sheets starting at the throat, on both sides of the diverging part. These sheets are subject to cloud cavitation detachments that are clearly not in phase, due to the bubbles' coalescence and the bubblescavitation interaction, as it can be seen in Fig. 8(a') and in the sequence of images in Fig. 9(a'). This may be due to the buoyancy force which is dominant and almost all of the injected bubbles interact with the cavitating zone on the upper wall. Furthermore, the cavitation is no more statistically symmetric (Fig. 10(a')). The injected air interacts with the upper cavitating zone and produces a large quantity of mixed vapour which spreads into multiple very small bubbles in the divergent zone of the venturi nozzle, as it can be seen in 
Fig. 8(a'). The dispersed bubbles are moreover trapped in a huge recirculation zone. Those bubble interact with the bottom cavitating cloud and influence its form. Nevertheless, a characteristic cavity length is still visible on the standard deviation plot in Fig. 10(a'). It is located on the bottom side between $2 H_{\text {throat }}$ and $3 H_{\text {throat }}$ (see the white arrow in Fig. 10(a') and the last instantaneous image in Fig. 9(a')). Moreover, the frequency of the cloud separation is changed, since the injected air makes the liquid passing through the throat "to push" the cloud towards the top wall of the venturi nozzle. This effect can be clearly seen in the sequence in Fig. 9(a').

A similar interaction is well present in Figs.9(b') and 10(b'), where the cavitation number has been decreased to the value of $\sigma=1.65$ and the cavitating zone has become larger. From the instantaneous images in Fig.9(b'), one can see that the bubbles expand their volume and influence the shape of the bottom cavitation zone. Those big bubbles further break up and mix with the cavitation vapour to form the dispersed black zone at the end of the divergent wall. This zone can be clearly identified in the standard deviation plot in Fig.10(b'). A characteristic length is difficult to be obtained.

On the other hand, at $\sigma=1.45$, the "supercavitation" can still be seen on Fig. 10(c'). One can see the tendency of the two liquid-vapour separation lines to bend towards the bottom. The injected bubbles grow rapidly due to the low pressure and expand their volume by reaching the walls on each side, as it can be seen in Figs. 8(c') and 9(c'). There is no cloud frequency separation that can be visually observed or detected. One can see the interaction between the expanded bubbles which cut the cavitating vapour on both sides of the venturi nozzle on the instantaneous snapshot in Fig. 8(c') and in more details in Fig. 9(c').

\subsubsection{Aerated cavitation time-space diagrams and frequency spectrums}

In Fig. 11 the time-space diagrams and frequency spectrum are plotted for the three aerated cases. For case (a'), the frequency spectrum is quite different compared to the one for pure cavitation (Fig. 6(a)). One can observe much more 

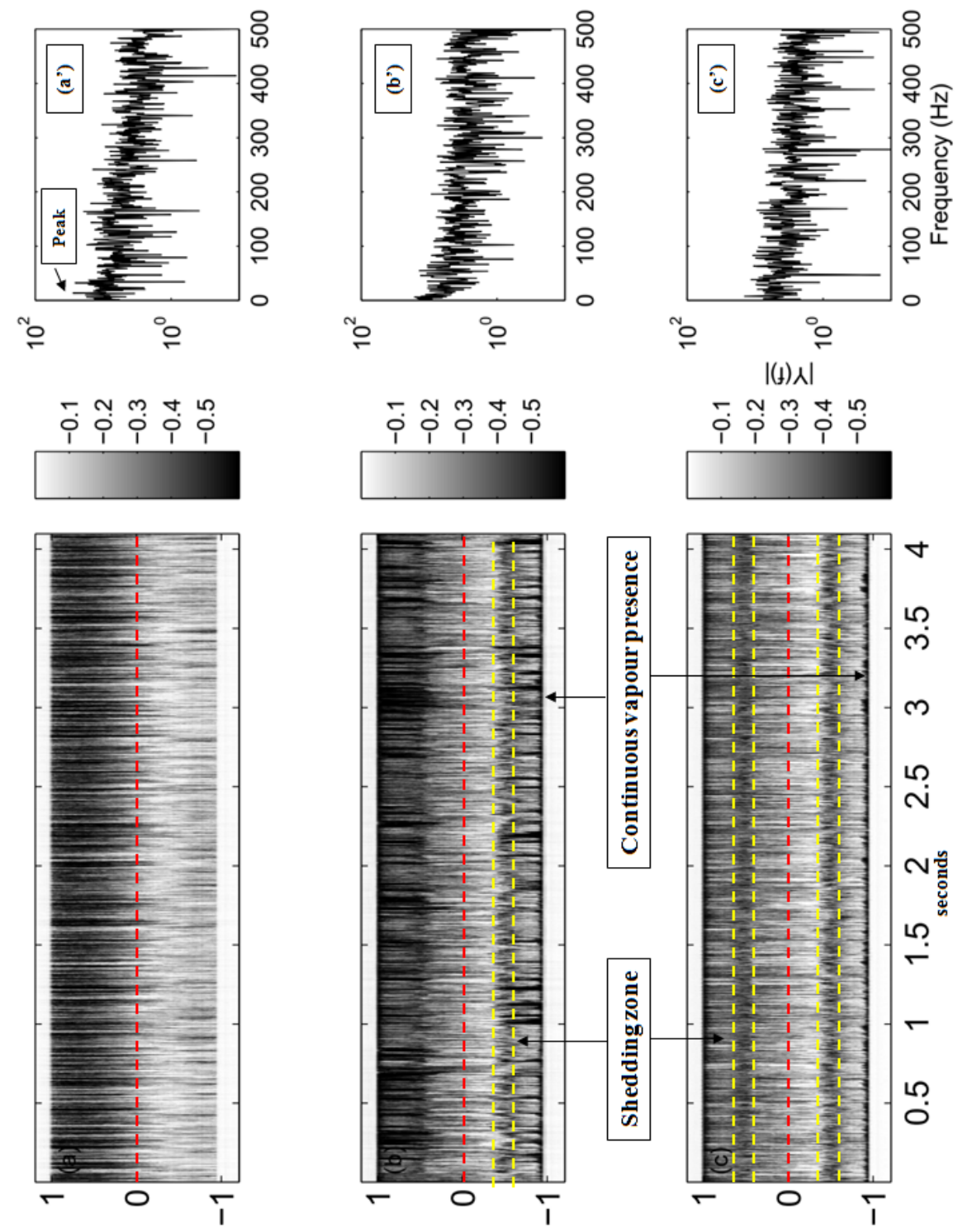

Figure 11: Aerated cavitation time-space diagrams and frequency spectrum along vertical line at $x^{*}=3.5$ : (a') $\sigma=1.74$; (b') $\sigma=1.65$; (c') $\sigma=1.45$.

peaks due to the injection of air which interacts with the cavitating vapour. In the range of 0 to $50 \mathrm{~Hz}$, four peak frequency values of $6 \mathrm{~Hz}, 10 \mathrm{~Hz}, 15 \mathrm{~Hz}$ and $32 \mathrm{~Hz}$ are observed. The first three peaks have almost the same amplitude. For 
higher frequencies, compared to the plot in Fig. 6(a), a peak level of $164 \mathrm{~Hz}$ is present instead of $145 \mathrm{~Hz}$. Furthermore, the time-space diagram is very different from the one without flow aeration. Indeed, the black stripes, as already mentioned, show the presence of bubbles inside the venturi nozzle along the monitored line. Almost all of the gaseous phase is located on the top wall in Fig. 11(a'). One can see there is no more symmetry on each of the walls in terms of the presence of the vapour, as was observed in Fig. 6(a). Moreover, the size of the vapour clouds is important, since the dark stripes go beyond the mean horizontal line of the axis. As a result, the cavitation zone on the bottom part is influenced by changing the nature of its expansion and its form.

On the other hand, in Fig. 11(b'), for $\sigma=1.65$, the frequency spectrum is more flat and equally spread than in Figs. 6(b) and 11(a'). Again, as in the cavitating cases, the frequency peak values disappear in the range of 0 to 50 $\mathrm{Hz}$ with the decrease of the cavitation number. It is interesting to observe the time-space diagram. One can see that there is almost only dark color on the top wall, which implies the huge presence of the air-vapour mixture. Another interesting inspection reveals that some of the dark strips connect without any discontinuities the top and bottom walls of the venturi nozzle in Fig. 11(b'). Such an observation implies that some of the flowing bubbles expand so much that they touch both walls without spreading apart. Another observation is the constant presence of discontinuities of the dark bottom lines in their vertical direction. Indeed, some of them change their color from dark to light. This is the result of the very rapid expansion of the injected bubbles which cut the developed cavitating cloud on the bottom and then mix with it in the form of small bubbles inside the divergent nozzle zone. Moreover, the cavitation does not have the time to develop in the same way as on Fig. 6(b).

The frequency spectrum at $\sigma=1.45$ is displayed in Fig. 11(c'). There is no clear frequency peak value, rather a continuous low-frequency part. What is also interesting is the fact that on the bottom wall there is an almost continuous black zone, which means that vapour is constantly present at the wall. There is not any trapped liquid between the vapour - liquid interface and the bottom 
wall. There is no symmetry with respect to the $\overrightarrow{\mathbf{x}}$. Many of the dark stripes connect the two ends of the nozzle. There is no physical meaning to calculate any Strouhal number for the cavitating vapour on the bottom venturi wall, since its shape is extremely dependent on the upstream flowing bubbles and their coalescence. Table 6 summarizes the gathered experimental data for each of the presented aerated cases.

\begin{tabular}{|c|c|c|c|}
\hline Case & $\sigma$ & $\operatorname{Str}_{L}$ & $\begin{array}{c}\mathbf{F} \\
(H z)\end{array}$ \\
\hline$\left(a^{\prime}\right)$ & 1.74 & - & 164 \\
\hline$\left(b^{\prime}\right)$ & 1.65 & - & 55 \\
\hline$\left(c^{\prime}\right)$ & 1.45 & - & - \\
\hline
\end{tabular}

Table 6: Aerated cavitation results data table

\section{Conclusion}

In the present paper, three different cavitation regimes have been studied: (a) cloud cavitation, (b) "quasi-supercavitation" and (c) "supercavitation". Those regimes have been further aerated by injecting air bubbles. The flow discharge velocity has been kept constant for all the flows, while the cavitation number has been decreased. The interaction between the top and bottom cavitating and aerated cavitatiting zones has been studied in the series of images showing the flow dynamics. Characteristic longitudinal lengths and characteristic frequencies have been extracted from statistics of the time series.

For the pure cavitation case at $\sigma=1.71$, the closure regions are cloud structures which are not connected by a vapour structure, while at $\sigma=1.46$ the two closure regions interact by a hairpin vortex. These regimes display periodical behaviors, with Strouhal numbers that correspond to values taken from the literature. When "supercavitation" regime at $\sigma=1.26$ is reached, the 
existence of shedding zones results in a trapped liquid-bubble mixture on the bottom wall. The bubbles flowing inside the trapped liquid are advected once they reach the shedding zone. The frequency spectrum suggests that no clear cloud separation could be observed in this regime. For the cases (a) and (b) the flow is statistically symmetrical, while for case (c) there is a slight tendency for the symmetry to be broken.

On the other hand, when the flows are aerated, the symmetry is immediately broken and characteristic lengths and frequencies are modified until a complete disappearance when "supercavitation" regime is reached. The pressure loss between the inlet and outlet of the venturi nozzle is greater than the one in pure cavitating case. At the same time, the visually observed flow behavior is quite different. The injected air bubbles, which are flowing in the middle section of the liquid zone, expand their volume. In such a way, at a certain moment, they break up into very small bubbles. Part of them are advected downstream the flow, while others follow the re-entrant jet at the cavity closure region that is present on the bottom side. The same bubbles create a vast and very well established recirculation zone at the end of the divergent part of the venturi nozzle for case (a'). As a result, the upper cavitating sheet is extremely disturbed by the bubble break up, and no periodical detachment can be observed for this upper sheet, while a periodic cycle is still present on the bottom side. In the case of aerated "supercavitation" (c'), the bubbles are unable to break apart, since their expansion reaches the top and bottom walls of the venturi nozzle, and are bounded by its geometry.

The smallest amount of injected air that could be reached in the present experiments is quite low, of the order of $1 \%$ by volume. It causes nevertheless drastic effects on the cavitation dynamics. It would be very interesting to reduce further the quantity of injected air in order to better characterize the transition from pure cavitation to aerated cavitation. These results will also be a benchmark for numerical modelings of aerated cavitation that are under development (Tomov et al., 2014). 


\section{Acknowledgment}

The authors would like to express their gratitude to Daniel Giroux for the exchanges during the writing of the article. The authors would also like to acknowledge the financial support granted by SNECMA, part of SAFRAN group.

\section{References}

Callenaere, M., Franc, J.P., Michel, J.M., Riondet, M., 2001. The cavitation instability induced by the development of a re-entrant jet. Journal of Fluid Mech. 444, 223-256.

Campos Amezcua, R., 2009. Analyse des écoulements cavitants stationnaires et instationnaires dans les turbomachines. Ph.D. thesis. Arts et Métiers ParisTech.

Ceccio, S.L., 2010. Friction Drag Reduction of External Flows with Bubble and Gas Injection. Ann. Rev. Fluid Mech. 42, 183-203.

Chen, G., Wang, G., Hu, C., Huang, B., Gao, Y., Zhang, M., 2015. Combined experimental and computational investigation of cavitation evolution and excited pressure fluctuation in a convergent-divergent channel. Int. J. Multiphase Flow 72, 133-140.

Coutier-Delgosha, O., Devillers, J.F., Pichon, T., Vabre, A., Woo, R., Legoupil, S., 2006. Internal structure and dynamics of sheet cavitation. Phys. Fluids $18,017103$.

Danlos, A., Méhal, J.E., Ravelet, F., Coutier-Delgosha, O., Bakir, F., 2014a. Study of the cavitating instability on a grooved venturi profile. J. Fluids Eng. $136,101302$.

Danlos, A., Ravelet, F., Coutier-Delgosha, O., Bakir, F., 2014b. Cavitation regime detection through Proper Orthogonal Decomposition: Dynamics analysis of the sheet cavity on a grooved convergentdivergent nozzle. Int. J. Heat and Fluid Flow 47, 9-20. 
Davis, M.P., 2008. Experimental Investigation of the Aviation Fuel Cavitation. Ph.D. thesis. University of Notre-Dame, Indiana.

Decaix, J., Goncalvès, E., 2013. Compressible effects modeling in turbulent cavitating flows. Eur. J. Mech. B 39, 11-31.

Dong, Z.Y., Su, P.L., 2006. Cavitation Control By Aeration and Its Compressible Characteristics. Journal of Hydrodynamics, Ser. B 18, 499-504.

Dular, M., Bachert, R., 2009. The issue of Strouhal number definition in cavitating flow. Journal of Mechanical Engineering 55, 666-674.

Dular, M., Bachert, R., Stoffel, B., Širok, B., 2005. Experimental evaluation of numerical simulation of cavitating flow around hydrofoil. Eur. J. Mech. B 24, $522-538$.

Dular, M., Khlifa, I., Fuzier, S., Adama Maiga, M., Coutier-Delgosha, O., 2012. Scale effect on unsteady cloud cavitation. Exp. In Fluids 53, 1233-1250.

Dular, M., Petkovšek, M., 2015. On the Mechanisms of Cavitation Erosion Coupling High Speed Videos to Damage Patterns. Exp. Thermal and Fluid Science 68, 359-370.

Dunn, P.F., Thomas, F.O., Davis, M.P., Dorofeeva, I.E., 2010. Experimental characterization of aviation-fuel cavitation. Phys. Fluids 22, 117102.

Fortes-Patella, R., Choffat, T., Reboud, J.L., Archer, A., 2013. Mass loss simulation in cavitation erosion: Fatigue criterion approach. Wear 300, 205-215.

Fortes-Patella, R., Reboud, J.L., 1998. A New Approach to Evaluate the Cavitation Erosion Power. J. Fluids Eng. 120, 335.

Fortes-Patella, R., Reboud, J.L., Archer, A., 2000. Cavitation damage measurement by 3D laser profilometry. Wear 246, 59-67.

Ganesh, H., Makiharju, S.A., Ceccio, S.L., 2014. Partial cavity shedding due to the propagation of shock waves in bubbly flows, in: Proceedings of the $30^{\text {th }}$ symposium on naval hydrodynamics. 
Gavaises, M., Villa, F., Koukouvinis, P., Marengo, M., Franc, J.P., 2015. Visualisation and les simulation of cavitation cloud formation and collapse in an axisymmetric geometry. Int. J. Multiphase Flow 68, 14-26.

Keil, T., Pelz, P.F., Buttenbender, J., 2012. On the transition from sheet to cloud cavitation, in: $8^{\text {th }}$ International Symposium on Cavitation, pp. 978-981.

Keil, T., Pelz, P.F., Cordes, U., Ludwig, G., 2011. Cloud Cavitation and Cavitation Erosion in Convergent Divergent Nozzle, in: WIMRC 3rd International Cavitation Forum.

Kjeldsen, M., Arndt, R.E.A., Effertz, M., 2000. Spectral Characteristics of Sheet/Cloud Cavitation. J. Fluids Eng. 122, 481-487.

Le, Q., Franc, J.P., Michel, J.M., 1993. Partial cavities: global behavior and mean pressure distribution. J. Fluids Eng. 115, 243-248.

Mejri, I., Bakir, F., Rey, R., Belamri, T., 2006. Comparison of Computational Results Obtained From a Homogeneous Cavitation Model With Experimental Investigations of Three Inducers. J. Fluids Eng. 128, 1308-1323.

Petkovšek, M., Dular, M., 2013. Simultaneous observation of cavitation structures and cavitation erosion. Wear 300, 55-64.

Pouffary, B., Fortes-Patella, R., Reboud, J.L., Lambert, P.A., 2008. Numerical Simulation of 3D Cavitating Flows: Analysis of Cavitation Head Drop in Turbomachinery. J. Fluids Eng. 130, 061301.

Savchenko, Y.N., 2001. Experimental Investigation of Supercavitating Motion of Bodies, in: RTO Educational Notes EN-010.

Sayyaadi, H., 2010. Instability of the cavitating flow in a venturi reactor. Fluid Dynamics Research 42, 055503.

Semenenko, V.N., 2001. Dynamic processes of supercavitation and computer simulation, in: RTO Educational Notes EN-010. 